\title{
Impact of proposed IASLC/ATS/ERS classification of lung adenocarcinoma: prognostic subgroups and implications for further revision of staging based on analysis of 514 stage I cases
}

\author{
Akihiko Yoshizawa ${ }^{1,2}$, Noriko Motoi ${ }^{1,3}$, Gregory J Riely ${ }^{4}$, Cami S Sima ${ }^{5}$, William L Gerald ${ }^{1, *}$, \\ Mark G Kris ${ }^{4}$, Bernard J Park ${ }^{6}$, Valerie W Rusch ${ }^{6}$ and William D Travis ${ }^{1}$ \\ ${ }^{1}$ Department of Pathology, Memorial Sloan-Kettering Cancer Center, New York, NY, USA; ${ }^{2}$ Department of \\ Laboratory Medicine, Shinshu University Hospital, Matsumoto, Japan; ${ }^{3}$ Department of Diagnostic Pathology, \\ the Cancer Institute Hospital of Japanese Foundation for Cancer Research, Tokyo, Japan; ${ }^{4}$ Thoracic Oncology \\ Service, Memorial Sloan-Kettering Cancer Center, New York, NY, USA; ${ }^{5}$ Epidemiology and Biostatistics, \\ Memorial Sloan-Kettering Cancer Center, New York, NY, USA and ${ }^{6}$ Thoracic Surgery Service, Memorial \\ Sloan-Kettering Cancer Center, New York, NY, USA
}

\begin{abstract}
A new lung adenocarcinoma classification is being proposed by the International Association for the Study of Lung Cancer, American Thoracic Society and European Respiratory Society (IASLC/ATS/ERS). This proposal has not yet been tested in clinical datasets to determine whether it defines prognostically significant subgroups of lung adenocarcinoma. In all, 514 patients who had pathological stage I adenocarcinoma of the lung classified according to the Union for International Cancer Control/American Joint Committee on Cancer 7th Edition, and who had undergone a lobectomy with mediastinal lymph node dissection were retrospectively reviewed. Comprehensive histological subtyping was used to estimate the percentage of each histological subtype and to identify the predominant subtype. Tumors were classified according to the proposed new IASLC/ATS/ERS adenocarcinoma classification. Statistical analyses were made including Kaplan-Meier and Cox regression analyses. There were 323 females $(63 \%)$ and 191 males $(37 \%)$ with a median age of 69 years (33-89 years) and 298 stage IA and 216 stage IB patients. Three overall prognostic groups were identified: low grade: adenocarcinoma in situ $(n=1)$ and minimally invasive adenocarcinoma $(n=8)$ had $100 \% 5$-year disease-free survival; intermediate grade: non-mucinous lepidic predominant $(n=29)$, papillary predominant $(n=143)$ and acinar predominant $(n=232)$ with 90,83 and $84 \%$ 5-year disease-free survival, respectively; and high grade: invasive mucinous adenocarcinoma $(n=13)$, colloid predominant $(n=9)$, solid predominant $(n=67)$ and micropapillary predominant $(n=12)$, with 75,7170 and $67 \%, 5$-year disease-free survival, respectively $(P<0.001)$. Among the clinicopathological factors, stage 1B versus 1A $(P<0.001)$, male sex $(P<0.008)$, high histological grade $(P<0.001)$, vascular invasion $(P=0.002)$ and necrosis $(P<0.001)$ were poorer prognostic factors on univariate analysis. Both gross tumor size $(P=0.04)$ and invasive tumor size adjusted by the percentage of lepidic growth $(P<0.001)$ were significantly associated with disease-free survival with a slightly stronger association for the latter. Multivariate analysis showed the prognostic groups of the IASLC/ATS/ERS histological classification $(P=0.038)$, male gender $(P=0.007)$, tumor invasive size $(P=0.026)$ and necrosis $(P=0.002)$ were significant poor prognostic factors. In summary, the proposed IASLC/ATS/ERS classification of lung adenocarcinoma identifies histological categories with prognostic differences that may be helpful in
\end{abstract}

Correspondence: Dr WD Travis, MD, Department of Pathology, Memorial Sloan-Kettering Cancer Center, 1275 York Avenue, New York, NY 10065, USA. E-mail: travisw@mskcc.org

Deceased.

Received 2 November 2010; revised 30 November 2010; accepted 30 November 2010; published online 21 January 2011 


\begin{abstract}
identifying candidates for adjunctive therapy. The slightly stronger association with survival for invasive size versus gross size raises the need for further studies to determine whether this adjustment in measuring tumor size could impact TNM staging for small adenocarcinomas.
\end{abstract}

Modern Pathology (2011) 24, 653-664; doi:10.1038/modpathol.2010.232; published online 21 January 2011 Keywords: adenocarcinoma; adenocarcinoma in situ; bronchioloalveolar carcinoma; lung; micropapillary;
minimally invasive adenocarcinoma; solid

Lung adenocarcinoma is a global public health problem as it is the most common histological subtype of lung cancer and worldwide lung cancer is the most common cause of major cancer incidence and mortality. ${ }^{1}$ Histological subclassification of lung adenocarcinoma has been a great challenge for many reasons. The tumor is very heterogeneous from every aspect: pathology, molecular, clinical, radiology and surgery. In the past decade numerous advances have taken place within each specialty for lung adenocarcinoma, particularly in molecular biology with the discovery that EGFR mutations are a marker for responsiveness to tyrosine kinase inhibitors. ${ }^{2,3}$ Pathological and radiological studies have also revealed significant prognostic subsets of lung adenocarcinoma. $^{4-6}$ However, neither the 2004 (WHO) World Health Organization nor the Noguchi classifications address very well the tumors with mixtures of histological types which result in classification of 90 and $60 \%$ of all resected lung adenocarcinomas as mixed subtype and as type $\mathrm{C}$, respectively., ${ }^{4,5}$ Because of these rapidly evolving advances in the field of lung adenocarcinoma, there is a need for improvement in stratification of histological categories according to prognosis compared with the previous classifications.

With this background, the International Association for the Study of Lung Cancer, American Thoracic Society and European Respiratory Society (IASLC/ATS/ERS), have sponsored a project to develop an International Multidisciplinary Lung Adenocarcinoma Classification. ${ }^{8}$ In this new classification (Table 1) there are several major changes for surgically resected tumors: (1) the term bronchioloalveolar carcinoma (BAC) is no longer used and the growth pattern of BAC is referred to as lepidic pattern; (2) adenocarcinoma in situ is proposed for small $(\leq 3 \mathrm{~cm})$ solitary adenocarcinomas with pure lepidic growth lacking invasion; (3) minimally invasive adenocarcinoma (MIA) is proposed for small $(\leq 3 \mathrm{~cm})$ lepidic predominant tumors with $\leq 0.5 \mathrm{~cm}$ of invasion; (4) invasive adenocarcinomas are now classified according to the predominant subtype after performing comprehensive histological subtyping with semiquantitative assessment of each subtype in 5\% increments; ${ }^{4}$ (5) micropapillary adenocarcinoma is added as a major subtype because of its poor prognostic significance in multiple studies of early stage lung adenocarcinoma; ${ }^{9-11}$ (6) former mucinous BACs are now classified as invasive mucinous adenocarcinomas recognizing that most of these tumors will have invasive
Table 12011 International Association for the Study of Lung Cancer, American Thoracic Society and European Respiratory Society international multidisciplinary classification of lung adenocarcinoma $^{\mathrm{a}}$

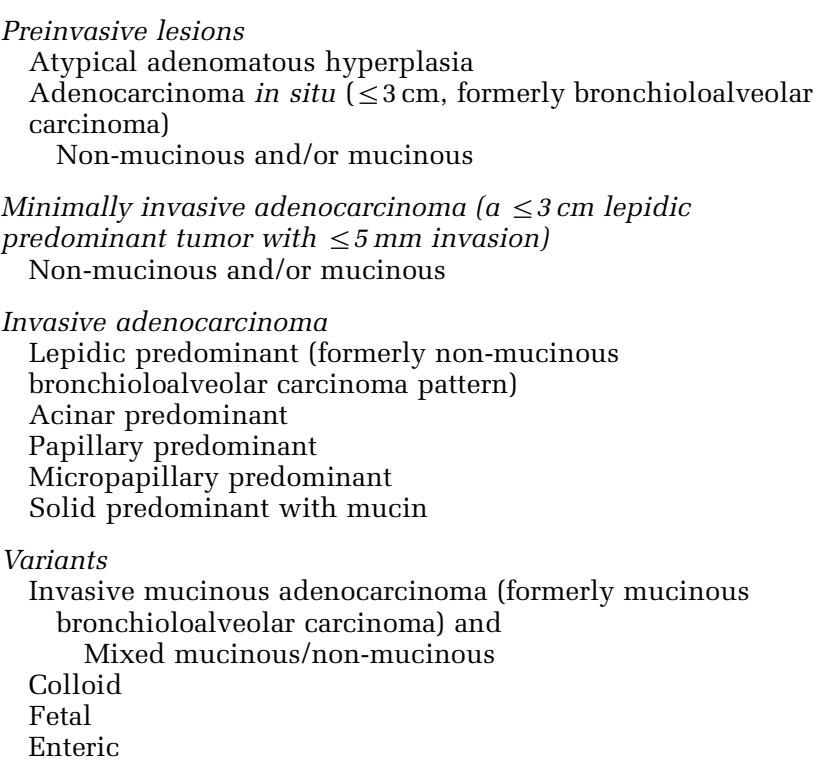

${ }^{\mathrm{a}}$ From Travis et al. ${ }^{8}$

components, less expression of TTF-1, frequent KRAS mutations, characteristic CT finding primarily of consolidation rather than ground glass opacities, worse prognosis than non-mucinous lepidic predominant adenocarcinomas and lack of responsiveness to tyrosine kinase inhibitors; ${ }^{12-19}$ and (7) clear cell and signet ring adenocarcinoma are recognized to represent cytological changes that occur in multiple histological subtypes rather than in separate histological subtype. ${ }^{20,21}$

We sought to explore the prognostic significance of this classification in a large series of surgically resected stage I lung adenocarcinomas. As most of these stage I patients did not receive neoadjuvant chemotherapy or adjuvant therapy, this study allowed for exploration of the prognostic significance of the proposed new histological subsets with minimal impact of chemotherapy.

\section{Patients and methods}

\section{Patients}

We evaluated a retrospective series of resected, preoperatively untreated stage I adenocarcinomas 
of the lung from Memorial Sloan-Kettering Cancer Center. Clinical data were retrieved from the Thoracic Surgical Database. This project was approved by the Memorial Sloan-Kettering Cancer Center institutional review board. These tumors were resected between January 1995 and November 2005. In all, 539 cases met the 2004 WHO criteria for adenocarcinoma and were considered as stage I according to the 6th edition Union for International Cancer Control/American Joint Committee on Cancer TNM classification and 25 cases were excluded after applying the new 7th Edition Union for International Cancer Control/American Joint Committee on Cancer TNM classification. ${ }^{22,23} \mathrm{~A}$ subset of 366 of these cases was previously reported to validate a grading system based on tumor architecture. $^{24}$

\section{Histological Evaluation}

All resected specimens were formalin fixed and stained with hematoxylin and eosin in the routine manner. As needed, mucin stains were performed to evaluate for mucin. Slides were evaluated microscopically by AY with problem cases reviewed by NM and WDT. The average number of slides from each case we reviewed in our study was 8.6 (range: 1-31).

Histological classification was according to the IASLC/ATS/ERS classification of lung adenocarcinomas and the 2004 WHO classification (Table 1). ${ }^{8}$ According to our previous paper by Motoi et al and the IASLC/ATS/ERS criteria, each tumor was reviewed using comprehensive histological subtyping, recording the percentage, in $5 \%$ increments, of each histological component. ${ }^{4}$ Tumors were classified as adenocarcinoma in situ (Figure 1), MIA (Figure 2), and invasive adenocarcinomas (Figure 3) which was further divided into lepidic predominant (Figure 3a), papillary predominant (Figure 3b), acinar predominant (Figure 3c), micropapillary predominant (Figure 3d), solid predominant (Figure 3e), invasive mucinous adenocarcinoma (Figure 4) and colloid predominant adenocarcinoma (Figure 5). The predominant pattern is defined as the pattern with the largest percentage, not necessarily $50 \%$ or greater. $^{4}$

Tumor size was measured according to 1) total tumor size and 2) size according to only the invasive component not including the lepidic growth. Total tumor size was assessed by standard gross measurement. Size of the invasive component was measured in two ways. In cases wherein the tumors were small, so the invasive component could be measured under the microscope on a single slide, the areas of invasion were measured at $\times 20$ or $\times 40$ magnification on an Olympus BX51 microscope using a ruler. However, in most cases wherein the tumor was large, and the size of invasion could not be measured on a single slide under the microscope, the total size of invasive tumor was calculated by multiplying the total size by the percentage of the invasive (non-lepidic) component.

We also investigated several histological factors, which have been reported as significant prognostic factors of lung adenocarcinoma: (1) visceral pleural invasion was classified as PL0, PL1 and PL2 according to the Union for International Cancer Control/American Joint Committee on Cancer TNM classification, 7 th Edition. ${ }^{25}$ (2) tumor grade of differentiation (well, moderately and poorly differentiated) was assessed according to the 2004 $\mathrm{WHO},{ }^{26}(3)$ vascular invasion and (4) necrosis was classified as present or absent.

\section{Statistics}

Overall survival was estimated using the KaplanMeier method, with patients followed from time
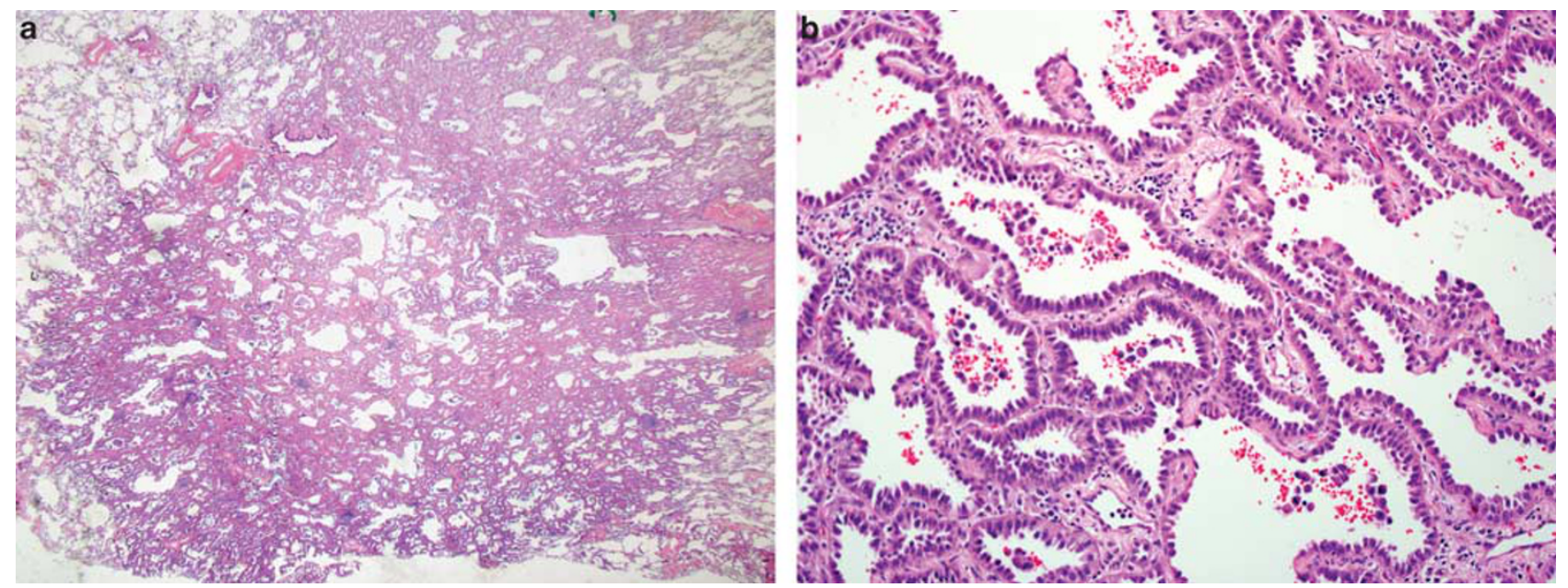

Figure 1 Adenocarcinoma in situ, non-mucinous. (a) This tumor shows a circumscribed nodule with lepidic growth consisting of alveolar wall thickening by atypical pneumocyte proliferation. No solid area of invasive growth is seen. (b) The pneumocytes show cellular crowding along the alveolar wall, with no invasion. 

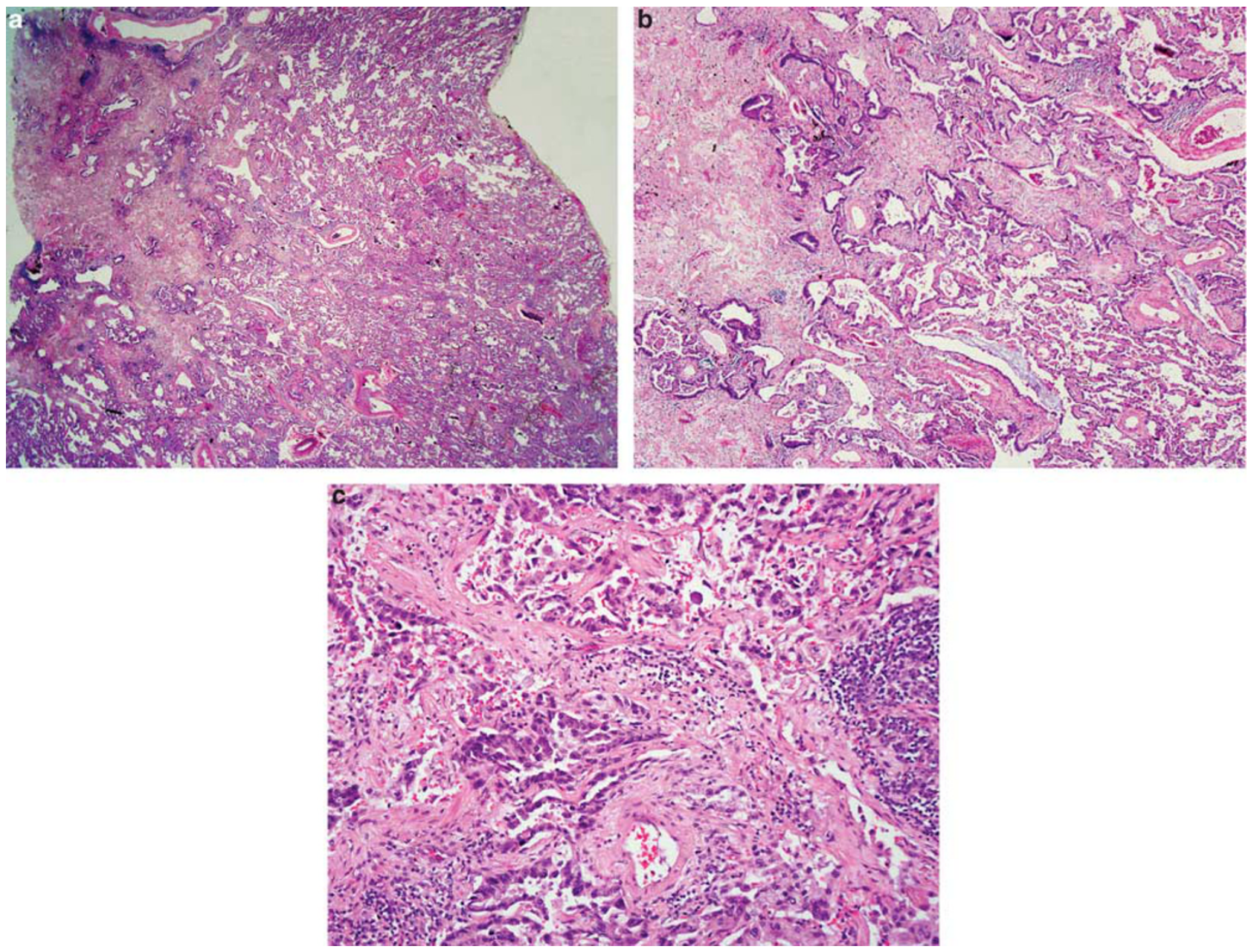

Figure 2 Minimally invasive adenocarcinoma, non-mucinous. (a) This tumor consists of mostly lepidic growth but there is a large subpleural scar with small foci of invasion at the edge of the scar. (b) At the edge of this scar are areas of invasive adenocarcinoma. (c) These malignant glands infiltrate desmoplastic stroma.

of surgery until death from any cause. Patients alive at the time of the last available follow-up were censored. Disease-free survival analysis followed patients until recurrence or death from disease. To account for the fact that some patients died of other causes, disease-free survival was evaluated using competing risk methods, which estimates the cumulative incidence function (CIF). Throughout the paper, disease-free survival results are reported as 1-CIF, for consistency with the more familiar Kaplan-Meier estimate. Univariate disease-free survival comparisons were performed using Gray's test, while the disease-free survival multivariate analysis used Cox proportional hazard model with patients censored at the time of death from other causes or at the last follow-up.

All statistical tests were two-sided and used 5\% level of significance. Statistical analysis was conducted using Statistical Analysis System (SAS) version 9.2 (SAS Institute Inc, Cary, NC, USA) and the cmprsk package in R (http://www.r-project.org/).

After reviewing the survival data, the histological subtypes were classified into three prognostic groups, which represented low, intermediate and high grades of clinical behavior. These prognostic groups actually revealed that the histological classification identifies categories of adenocarcinoma that have different grades of behavior. Therefore, this 'tumor grade' was based purely on the results of our survival analysis according to the major histological classification of the tumor and differed from the traditional 'differentiation grade' according to the 2004 WHO criteria. ${ }^{26}$

\section{Results}

\section{Clinical Characteristics}

Clinical features including age, sex, race, smoking status and stage are summarized according to histological subtype in Table 3 . Most patients were female $(63 \%)$ and stage IA (58\%). Median age was 69 years (range: 33-89 years) and median tumor size was $2.0 \mathrm{~cm}$ (range: $0.3-5 \mathrm{~cm}$ ). In all, 301 tumors $(59 \%)$ were located in right lung and 213 tumors $(41 \%)$ were located in left lung. One patient 

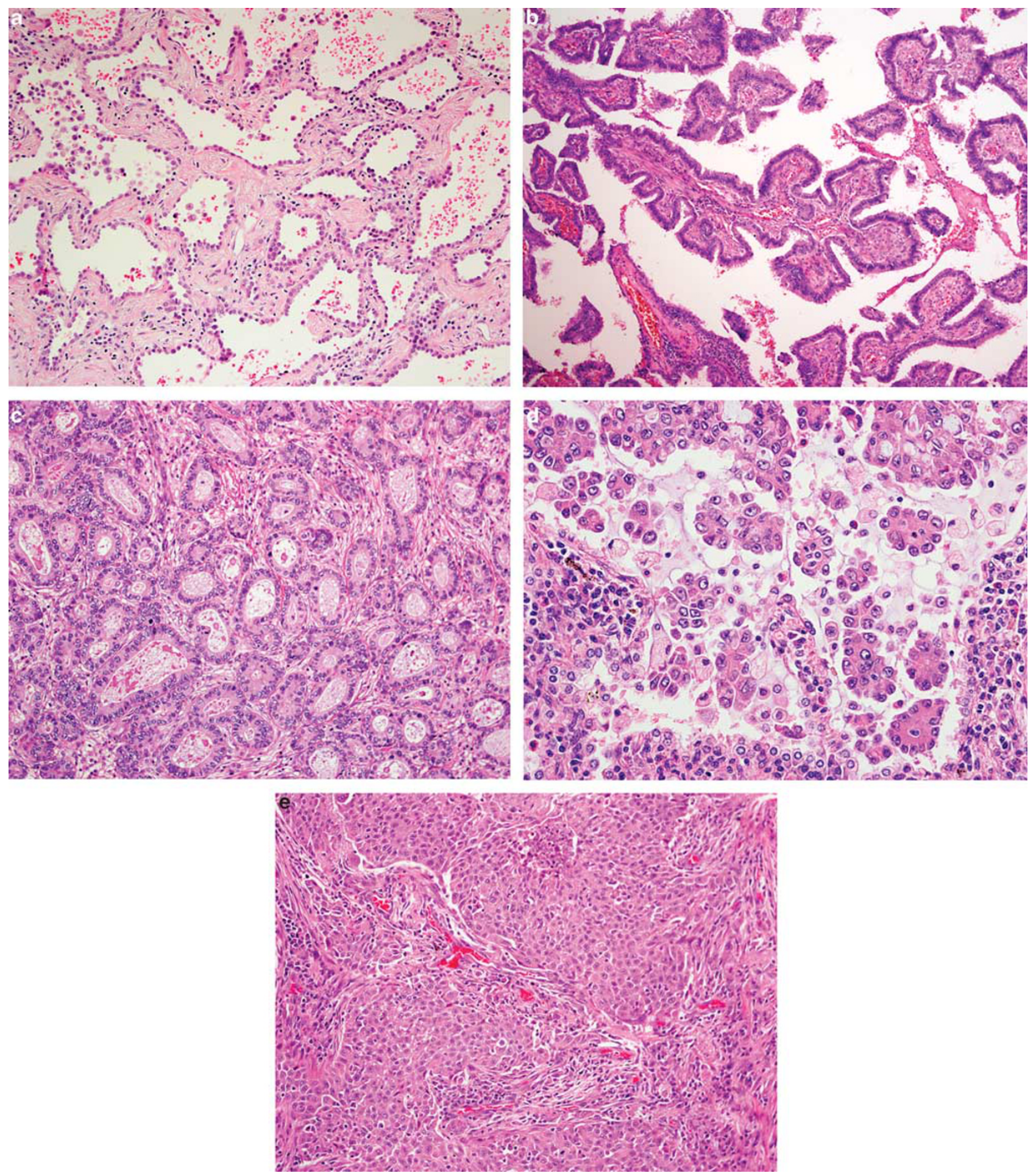

Figure 3 Patterns of invasive adenocarcinoma. (a) Lepidic, this tumor shows a non-mucinous lepidic pattern with crowded atypical pneumocytes proliferating along the surface of slightly thickened alveolar walls. No invasion is seen. (b) Papillary, this tumor shows a papillary pattern with cuboidal tumor cells growing along the surface of fibrovascular cores. (c) Acinar, this tumor is composed of round to oval-shaped glands consisting of atypical epithelial cells showing nuclear hyperchromasia and prominent nucleoli. (d) Micropapillary, this tumor shows glandular cells growing in small papillary tufts lacking fibrovascular cores. (e) Solid, this tumor is composed of solid sheets of tumor cells.

underwent pneumonectomy, 3 patients underwent bilobectomy, 429 patients $(83 \%)$ underwent lobectomy, 31 patients $(6 \%)$ underwent segmentectomy and 50 patients $(10 \%)$ underwent wedge resection.
2004 WHO histological grade was well, moderate and poorly differentiated in 58 (11\%), 287 (56\%) and $169(33 \%)$ of cases. Necrosis and vascular invasion were seen in $97(19 \%)$ and $123(24 \%)$ of 

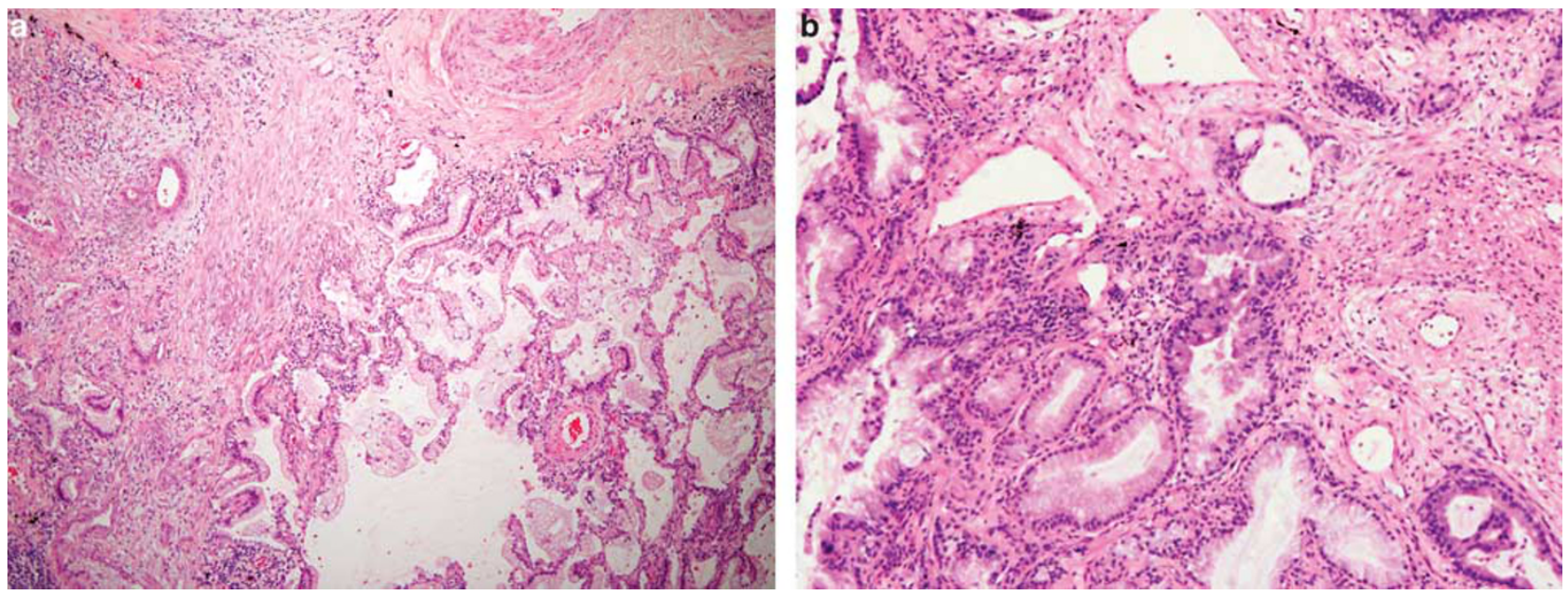

Figure 4 Mucinous adenocarcinoma. (a) This mucinous adenocarcinoma shows lepidic growth along the alveolar walls, and also invasive acinar growth with penetration of fibrous stroma. (b) This area of the mucinous tumor shows an acinar pattern of invasion.

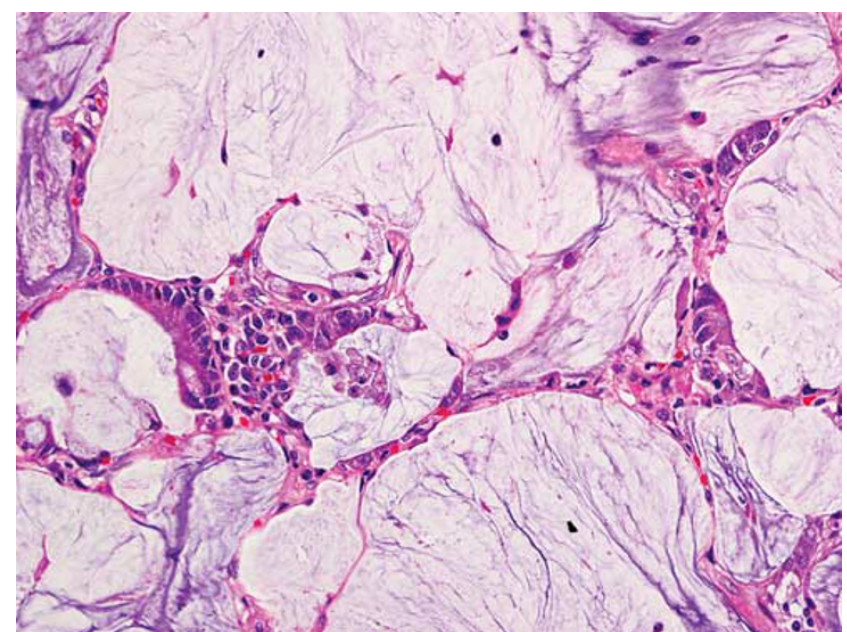

Figure 5 Colloid adenocarcinoma. This tumor consists of pools of mucin filling air spaces with well-differentiated malignant glands growing along the edges of the surrounding connective tissue.

the cases, respectively. Visceral pleural invasion (PL1 or PL2) was seen in 103 cases $(20 \%){ }^{25}$

No patient received neoadjuvant therapy; adjuvant chemotherapy and/or radiation therapy was administered in 8 patients $(2 \%)$ with unknown adjuvant therapy status in $20(4 \%)$.

\section{Pathological Characteristics}

The distribution of histological subtypes according to the 2004 WHO and the IASLC/ATS/ERS classifications is summarized in Table 2. According to the 2004 WHO classification, 95\% of the cases were classified as mixed subtype. Only one tumor was classified as non-mucinous BAC or adenocarcinoma in situ according to the 2004 WHO and IASLC/ATS/ ERS classifications, respectively.

According to the IASLC/ATS/ERS classification, seven of the eight MIA cases were non-mucinous and the eighth case with mucinous features also had a minor non-mucinous component, but no cases of mucinous adenocarcinoma in situ were seen in this study. For the 29 lepidic predominant non-mucinous tumors, the percent lepidic growth averaged $55 \%$ (range: $40-90 \%$ ) and in seven cases it was only $40 \%$. For the 12 mucinous adenocarcinomas, the percent lepidic growth averaged 57\% (range: $40-80 \%$ ) and in two cases there was only $40 \%$ lepidic growth, so there was $60 \%$ invasion.

The greatest impact of the adjustment of mean tumor size from total gross measurement to mean invasive tumor size (Table 3) was with MIA (1.3-0.2 $\mathrm{cm}, 84 \%$ reduction), lepidic predominant $(2.3-1 \mathrm{~cm}, 57 \%$ reduction) and mucinous adenocarcinoma $(2.5-1.1 \mathrm{~cm}, 56 \%$ reduction).

After adjustment for the percentage of lepidic growth, T-size factor status was downgraded from T1b to T1a in 52 cases, from T2a to T1a in 9 cases and from T2a to T1b in 11 cases. Thus T-factor status was decreased in 72 patients $(14 \%)$ of the total cohort. When this method was used to determine TNM staging, 3 of 25 cases that were excluded from the total 539 cases would have been downstaged from stage IIA to stage IB, However these cases were not included in this study as they do not meet the Union for International Cancer Control/American Joint Committee on Cancer 7 th Edition stage 1 criteria.

\section{Survival Analysis}

In all, 70 patients (14\%) died of disease and 105 $(21 \%)$ died of other causes or unknown cause during the study follow-up. Of the remaining $320(62 \%)$ patients alive at the time of analysis, 12 had recurrent disease, 4 were diagnosed with new lung cancers and 304 had no evidence of disease. A total of 89 patients recurred or died from disease, counting as events in the disease-free survival 
Table 2 Adenocarcinoma subtypes by International Association for the Study of Lung Cancer, American Thoracic Society and European Respiratory Society and 2004 World Health Organization classifications

\begin{tabular}{|c|c|c|c|}
\hline IASLC/ATS/ERS & Number (\%) & $2004 \mathrm{WHO}$ & Number (\%) \\
\hline NA & & Mixed subtype (MS) & $490(95)$ \\
\hline Adenocarcinoma in situ & $1(0.2)$ & BAC, non-mucinous & $1(0.2)$ \\
\hline Minimally invasive adenocarcinoma, non-mucinous & $7(1.4)$ & NA (included in mixed subtype) & 0 \\
\hline MIA, mixed mucinous and non-mucinous & $1(0.2)$ & NA (included in mixed subtype) & 0 \\
\hline \multicolumn{4}{|l|}{ Invasive adenocarcinoma } \\
\hline Lepidic predominant & $29(5.6)$ & NA (included in mixed subtype) & 0 \\
\hline Acinar predominant & $232(45.1)$ & Acinar & $6(1.2)$ \\
\hline Papillary predominant & $143(27.8)$ & Papillary & $11(2.1)$ \\
\hline Micropapillary predominant & $12(2.3)$ & NÁ & 0 \\
\hline Solid predominant & $67(13)$ & Solid & $1(0.2)$ \\
\hline Variants & & & \\
\hline $\begin{array}{l}\text { Invasive mucinous adenocarcinoma } \\
\text { (and mixed mucinous/non-mucinous) }\end{array}$ & $13(2.5)$ & No pure M BAC (included in mixed subtype) & 0 \\
\hline Colloid predominant & $9(1.8)$ & Colloid & $4(0.8)$ \\
\hline NA & 0 & Signet ring & $1(0.2)$ \\
\hline
\end{tabular}

Abbreviations: NA, not applicable; WHO, World Health Organization.

Table 3 Clinical features according to IASLC/ATS/ERS histological subtype

\begin{tabular}{|c|c|c|c|c|c|c|c|c|}
\hline Histological subtype & $\begin{array}{c}\mathrm{N} \\
(\%)\end{array}$ & $\begin{array}{l}\text { Mean } \\
\text { age yrs } \\
\text { (range) }\end{array}$ & $\begin{array}{c}S e x \\
(M / F)\end{array}$ & $\begin{array}{l}\text { Race } \\
W / O\end{array}$ & $\begin{array}{c}\text { Smoking } \\
C / F / N(M P Y)\end{array}$ & $\begin{array}{l}\text { Stage } \\
1 A / B\end{array}$ & $\begin{array}{c}\text { Mean gross } \\
\text { size } \mathrm{cm} \\
\text { (range) }\end{array}$ & $\begin{array}{c}\text { Mean } \\
\text { invasive } \\
\text { size } \mathrm{cm} \\
\text { (range) }\end{array}$ \\
\hline AIS or MIA ${ }^{a}$ & 9 & $68(52-81)$ & $4 / 5$ & $6 / 3$ & 0/8/1 (23) & $9 / 0$ & $1.3(0.5-2)$ & $0.2(0-0.3)$ \\
\hline Lepidic predominant & $29(5.6)$ & $69(43-84)$ & $17 / 12$ & $28 / 1$ & $1 / 22 / 5(19)$ & $17 / 12$ & $2.3(0.9-4.6)$ & $1(0.3-2.8)$ \\
\hline Acinar predominant & $232(45.1)$ & $68(33-89)$ & $71 / 161$ & $209 / 23$ & 29/158/45 (36) & $138 / 94$ & $2.1(0.3-5.0)$ & $1.9(0.3-5.0)$ \\
\hline Papillary predominant & $143(27.8)$ & $67(42-87)$ & $55 / 88$ & $130 / 13$ & 20/104/18 (46) & $88 / 55$ & $2.2(0.7-5.0)$ & $1.9(0.6-5.0)$ \\
\hline Micropapillary predominant & $12(2.3)$ & $72(61-86)$ & $6 / 6$ & $12 / 0$ & $2 / 7 / 3(38)$ & $6 / 6$ & $2.8(1.5-5.0)$ & $2.8(1.5-5.0)$ \\
\hline Solid predominant & $67(13)$ & $66(43-83)$ & $29 / 38$ & $62 / 5$ & 15/50/1 (59) & $28 / 39$ & $2.5(0.5-5.0)$ & $2.5(0.5-5.0)$ \\
\hline $\begin{array}{l}\text { Invasive mucinous and mixed } \\
\text { mucinous/non-mucinous }\end{array}$ & $13(2.5)$ & $71(54-85)$ & $4 / 9$ & $13 / 0$ & $5 / 5 / 3(37)$ & $7 / 6$ & $2.5(0.5-3.6)$ & $1.1(0.3-1.8)$ \\
\hline $\begin{array}{l}\text { Adenocarcinoma } \\
\text { Colloid predominant }\end{array}$ & $9(1.8)$ & $62(49-77)$ & $5 / 4$ & $9 / 0$ & $3 / 4 / 2(38)$ & $5 / 4$ & $2.5(1.3-5.0)$ & $2.3(1.0-5.0)$ \\
\hline Total & 514 & $68(33-89)$ & $\begin{array}{l}191(37 \%) / \\
323(63 \%)\end{array}$ & $\begin{array}{c}469(91 \%) / \\
45(9 \%)\end{array}$ & $\begin{array}{r}75(15 \%) / \\
358(70 \%) / \\
78(15 \%)^{b} \\
(41)\end{array}$ & $\begin{array}{c}298 \\
(58 \%) / \\
216 \\
(42 \%)\end{array}$ & $2.2(0.3-5.0)$ & $1.9(0-5.0)$ \\
\hline
\end{tabular}

Abbreviations: AIS, adenocarcinoma in situ; ATS, American Thoracic Society; C, current; ERS, European Respiratory Society; F, former; IASLC, International Association for the Study of Lung Cancer; MIA, minimally invasive adenocarcinoma; MPY, mean pack years; N, never; O, other; W, white.

${ }^{\mathrm{a}}$ This category includes: 1 AIS, 7 non-mucinous MIA and 1 mixed mucinous/non-mucinous MIA

${ }^{\mathrm{b}}$ Three patients with unknown smoking status.

analysis. Median clinical follow-up was 4 years (range: 0.02-13.3 years).

With $95 \%$ of the patients classified into mixed group, the WHO classification did not provide a useful stratification with respect to disease-free survival $(P=0.48)$.

According to disease-free survival, three overall prognostic groups with low, intermediate and highgrade clinical behavior were identified (Table 4, Figure $6 a$ and $b$ ). Low grade comprised of adenocarcinoma in situ $(n=1)$ and MIA $(n=8)$, both with $100 \%$ disease-free survival at 5 years. Intermediate-grade tumors consisted of non-mucinous lepidic predominant $(n=29$, disease-free survival at
$5 \mathrm{yrs}=90 \%)$, acinar predominant $(n=232$, diseasefree survival at $5 \mathrm{yrs}=84 \%$ ) and papillary predominant $(n=143$, disease-free survival at $5 \mathrm{yrs}=83 \%)$ adenocarcinomas. High-grade tumors included: solid predominant $(n=67$, disease-free survival at $5 \mathrm{yrs}=70 \%)$, micropapillary predominant $(n=12$, disease-free survival at $5 \mathrm{yrs}=67 \%$ ), colloid predominant $(n=9$, disease-free survival at $5 \mathrm{yrs}=71 \%)$ and invasive mucinous and mixed mucinous/ non-mucinous $(n=13$, disease-free survival at $5 \mathrm{yrs}=76 \%$ ).

There was no significant difference with respect to disease-free survival between any two histological subtypes belonging to the same risk group. 
Table 4 Survival according to IASLC/ATS/ERS adenocarcinoma histological subtypes

\begin{tabular}{|c|c|c|}
\hline $\begin{array}{l}\text { IASLC/ATS/ERS classification } \\
\text { subtypes }\end{array}$ & $\begin{array}{l}\text { Number } \\
(\%)\end{array}$ & $\begin{array}{l}\text { Disease-free } \\
\text { survival at } 5 \text { years }\end{array}$ \\
\hline \multicolumn{3}{|l|}{ Low grade } \\
\hline Adenocarcinoma in situ & $1(0.2 \%)$ & $100 \%$ \\
\hline $\begin{array}{l}\text { Minimally invasive } \\
\text { adenocarcinoma, non-mucinous }\end{array}$ & $7(1 \%)$ & $100 \%$ \\
\hline $\begin{array}{l}\text { Minimally invasive } \\
\text { adenocarcinoma, mixed } \\
\text { mucinous and non-mucinous }\end{array}$ & $1(0.2 \%)$ & $100 \%$ \\
\hline \multicolumn{3}{|l|}{ Intermediate grade } \\
\hline Lepidic predominant & $29(6 \%)$ & $90 \%$ \\
\hline Acinar predominant & $232(45 \%)$ & $84 \%$ \\
\hline Papillary predominant & $143(28 \%)$ & $83 \%$ \\
\hline \multicolumn{3}{|l|}{ High grade } \\
\hline Micropapillary predominant & $12(2 \%)$ & $67 \%$ \\
\hline Solid predominant & $67(13 \%)$ & $70 \%$ \\
\hline Colloid predominant & $9(2 \%)$ & $71 \%$ \\
\hline $\begin{array}{l}\text { Invasive mucinous } \\
\text { adenocarcinoma, mixed } \\
\text { mucinous/non-mucinous }\end{array}$ & $13(3 \%)$ & $76 \%$ \\
\hline
\end{tabular}

Abbreviations: ATS, American Thoracic Society; ERS, European Respiratory Society; IASLC, International Association for the Study of Lung Cancer.

Disease-free survival estimates (1-CIF or cumulative index function) are calculated based on the competing risk analysis.
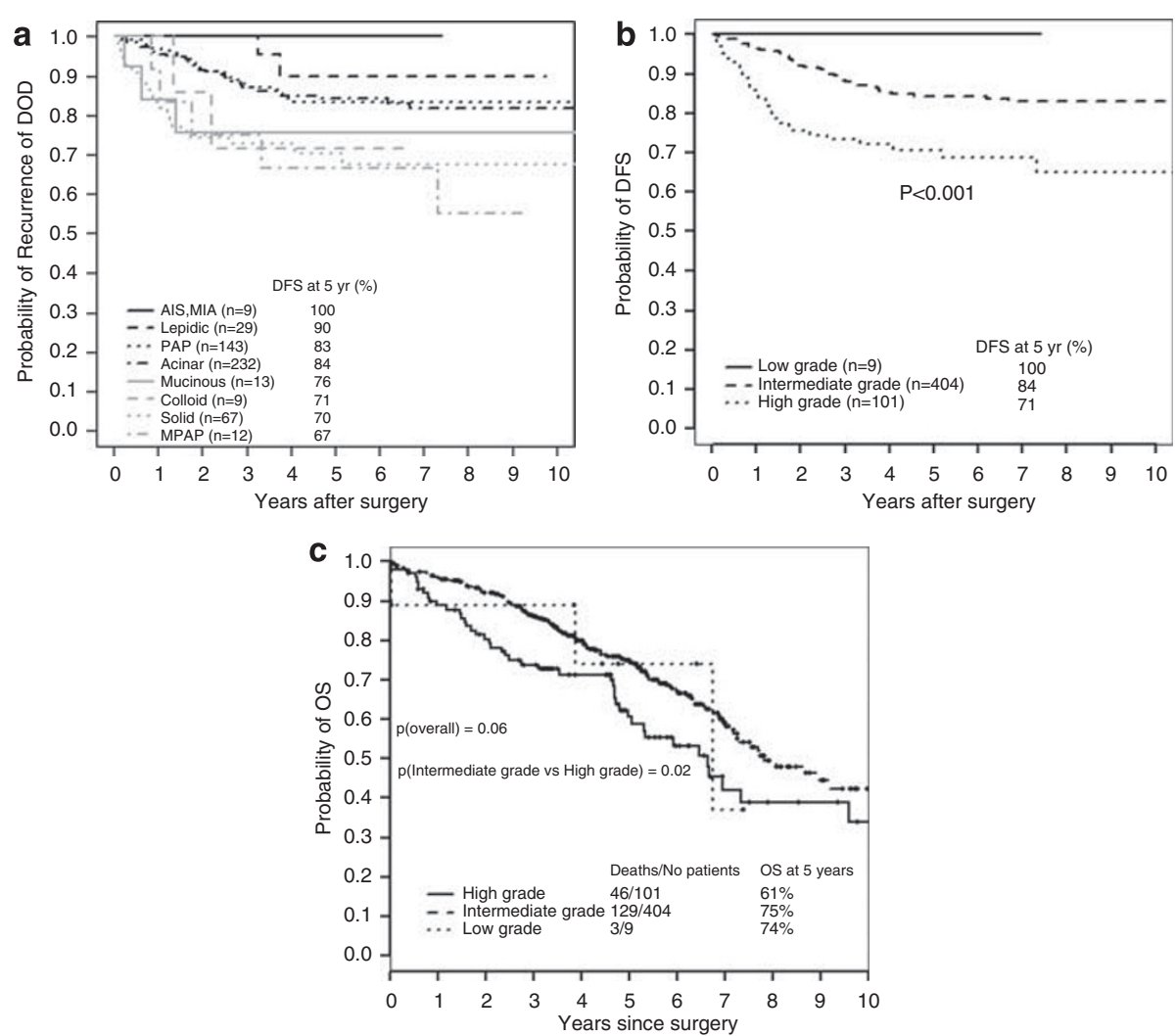

Figure 6 (a) Disease-free survival (DFS) for all histological categories $(P<0.001)$. The favorable group includes adenocarcinoma in situ (AIS) and minimally invasive adenocarcinomas (MIA) with 100\% 5-year disease-free survival. Disease-free survival for the intermediate group was 90, 83 and $84 \%$ for lepidic predominant, papillary (PAP) predominant and acinar predominant, and adenocarcinomas, respectively. Disease-free survival for the unfavorable group was $70,67,71$ and $76 \%$ for solid predominant, micropapillary (MPAP) predominant colloid predominant, and mucinous and mixed adenocarcinomas, respectively. (b) Disease-free survival according to combined histological groupings according to low, intermediate and high-grade clinical aggressiveness. (c) Overall survival (OS) according to combined histological groupings according to low, intermediate and high-grade clinical aggressiveness.

Considering this, as well as the small samples sizes observed for most of the histological subtypes, we further conducted survival analysis according to low $(n=9)$, intermediate $(n=404)$ and high-grade $(n=101)$ groupings and found 5-year disease-free survival of 100,84 and $71 \%$, respectively $(P<0.001)$. Table 5 summarizes the univariate association between clinical factors and disease-free survival. Higher stage $(P<0.001)$, male gender $(P=0.008)$, necrosis $(P<0.001)$, poor differentiation $(P<0.001)$ and vascular invasion $(P=0.002)$ were all ssociated with worse disease-free survival.

For overall survival, the three grading categories determined by IASLC/ATS/ERS classification were overall survival, dikely becent $(P=0.06)$ with respect to patients, lack of events in the low-grade group and death due to other causes. However, patients with intermediate grade had significantly better overall survival, compared with high-grade patients $(P=0.02)$

According to the Union for International Cancer Control/American Joint Committee on Cancer, TNM classification, disease-free survival for patients with T1a $(\leq 2 \mathrm{~cm})$ tumors was significantly better compared with that for $\mathrm{T} 1 \mathrm{~b}(>2 \mathrm{~cm}$ or $\leq 3 \mathrm{~cm})$ tumors

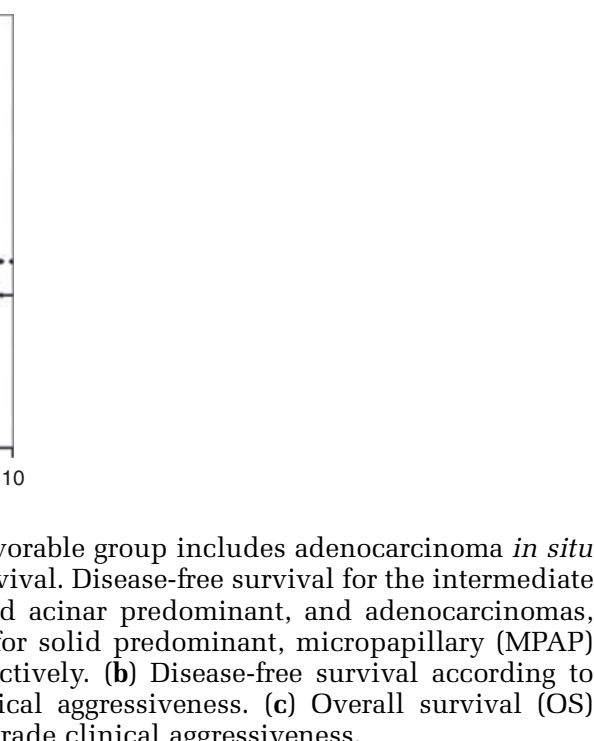


Table 5 Association between clinical factors and disease-free survival

\begin{tabular}{|c|c|c|c|}
\hline Factor & Number (\%) & 5-year DFS & P-value \\
\hline \multicolumn{4}{|l|}{ Stage } \\
\hline IA & $298(58 \%)$ & $88 \%$ & \multirow[t]{2}{*}{$<0.001$} \\
\hline IB & $216(42 \%)$ & $74 \%$ & \\
\hline \multicolumn{4}{|l|}{ Sex } \\
\hline Female & $323(63 \%)$ & $86 \%$ & \multirow[t]{2}{*}{0.008} \\
\hline Male & $191(37 \%)$ & $76 \%$ & \\
\hline \multicolumn{4}{|l|}{ Grade } \\
\hline Well/moderate & $345(67 \%)$ & $87 \%$ & \multirow[t]{2}{*}{$<0.001$} \\
\hline Poor & $169(33 \%)$ & $72 \%$ & \\
\hline \multicolumn{4}{|l|}{ Necrosis } \\
\hline No & $416(81 \%)$ & $87 \%$ & \multirow{2}{*}{$<0.001$} \\
\hline Yes & $97(19 \%)$ & $63 \%$ & \\
\hline \multicolumn{4}{|c|}{ Visceral pleural invasion } \\
\hline PL0\&1 & $411(80 \%)$ & $83 \%$ & \multirow[t]{2}{*}{0.09} \\
\hline PL2 & $103(20 \%)$ & $77 \%$ & \\
\hline \multicolumn{4}{|l|}{ Vascular invasion } \\
\hline No & $391(76 \%)$ & $85 \%$ & \multirow[t]{2}{*}{0.002} \\
\hline Yes & $123(24 \%)$ & $74 \%$ & \\
\hline \multicolumn{4}{|l|}{ Race } \\
\hline White & $469(91 \%)$ & $82 \%$ & \multirow{2}{*}{0.97} \\
\hline Other & $45(9 \%)$ & $82 \%$ & \\
\hline \multicolumn{4}{|l|}{ Ever smoker } \\
\hline No & $78(15 \%)$ & $90 \%$ & \multirow[t]{2}{*}{0.07} \\
\hline Yes & $433(84 \%)$ & $81 \%$ & \\
\hline
\end{tabular}

Abbreviation: DFS, disease-free survival.

DFS estimates (1-CIF or cumulative index function) are calculated based on the competing risk analysis.

$(P=0.04$; Figure 7a), and the difference became even more pronounced when size was adjusted for the amount of invasive size $(P<0.001$, Figure $7 \mathrm{~b})$.

\section{Cox Regression}

A multivariate analysis adjusting for gender, stage, tumor size, differentiation, presence of necrosis and of vascular invasion indicated that histological grading remained significantly associated with disease-free survival (Table 6), with patients in the high-risk group having an increased risk of death, compared with the intermediate/low-risk group and to the intermediate-risk group alone $(\mathrm{HR}=1.68$, 95\% CI: $1.03-2.75, P=0.038$, for both comparisons). Other independent predictors of an increased risk of death of disease or recurrence were: male gender $(\mathrm{HR}=1.8, P=0.007)$, tumor size adjusted for lepidic growth $(\mathrm{HR}=1.3, P=0.026)$ and tumor necrosis $(\mathrm{HR}=1.5, P=0.002)$.

\section{Discussion}

Our data show the proposed IASLC/ATS/ERS classification identifies histological subsets of lung
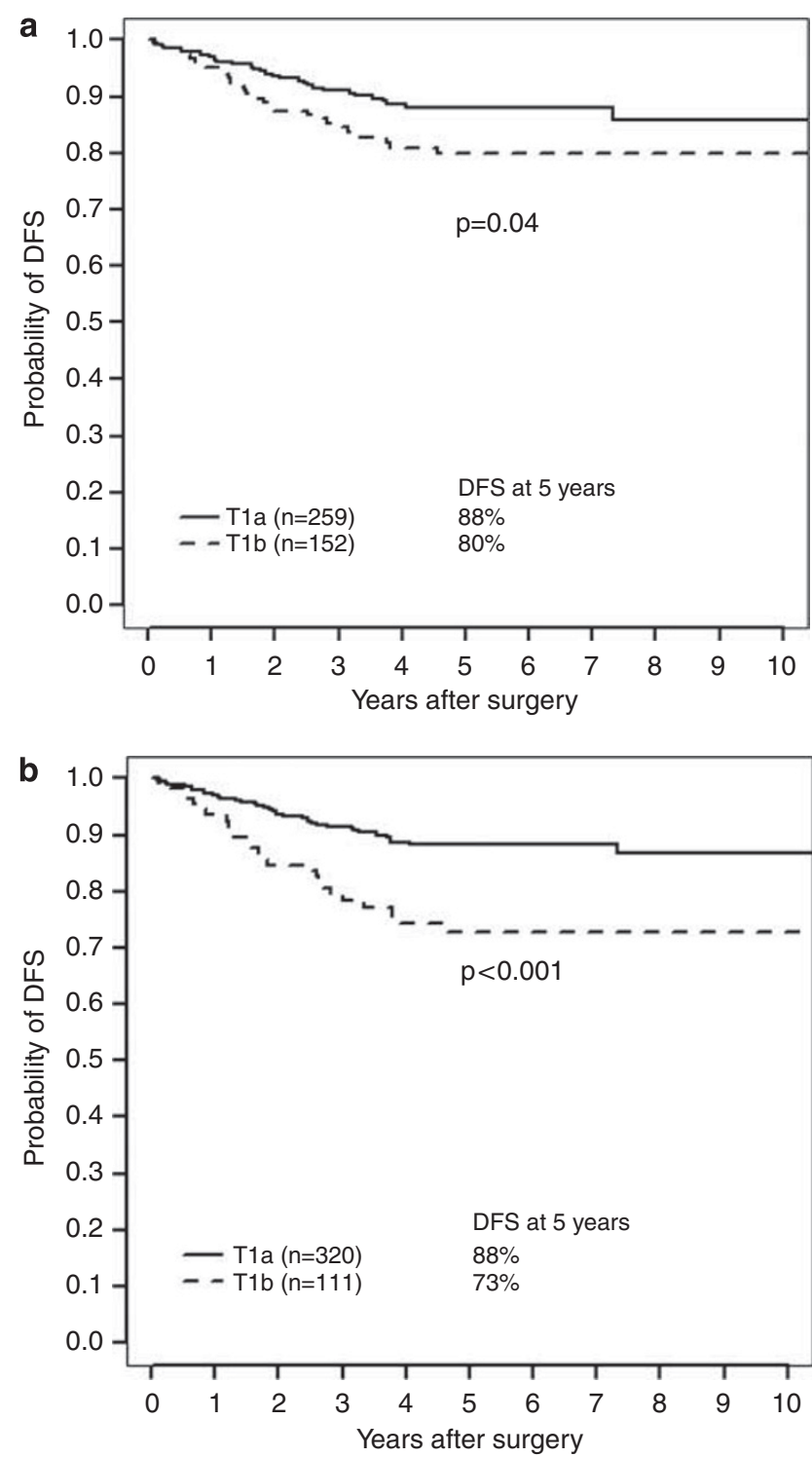

Figure 7 Disease-free survival (DFS) comparing T1a $(\leq 2 \mathrm{~cm})$ versus $\mathrm{T} 1 \mathrm{~b}(>2 \mathrm{~cm}$ or $\leq 3 \mathrm{~cm}$ ). (a) T1a and T1b defined according to gross tumor size $(P=0.04)$. (b) T1a and T1b defined according to invasive size $(P<0.001)$.

Table 6 Multivariate survival analysis for disease-free survival

\begin{tabular}{lcc}
\hline Factor & HR (95\% CI) & P-value \\
\hline $\begin{array}{l}\text { Tumor grade by IASLC/ATS/ERS } \\
\text { classification (high versus }\end{array}$ & $1.7(1.03-2.75)$ & 0.038 \\
$\begin{array}{l}\text { intermediate/low) } \\
\text { Gender (male versus female) }\end{array}$ & $1.79(1.17-2.74)$ & 0.007 \\
$\begin{array}{l}\text { Stage (IB versus IA) } \\
\text { Tumor size }\end{array}$ & $1.40(0.84-2.33)$ & 0.19 \\
$\begin{array}{l}\text { 2004 WHO histological grade } \\
\text { (poor versus moderate/well) }\end{array}$ & $1.29(1.03-1.62)$ & 0.026 \\
$\begin{array}{l}\text { Necrosis (yes versus no) } \\
\text { Vascular invasion (yes versus no) }\end{array}$ & $1.159(1.62-1.78)$ & 0.86 \\
& & \\
\end{tabular}

Abbreviations: ATS, American Thoracic Society; ERS, European Respiratory Society; IASLC, International Association for the Study of Lung Cancer; WHO, World Health Organization.

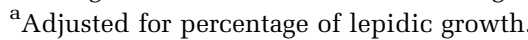


adenocarcinoma with significant differences in prognosis. As patients with surgically resected stage I non-small cell lung cancer have survivals of $73 \%$ for stage IA and $58 \%$ for stage $\mathrm{IB},{ }^{27}$ there is a great need to predict which patients will recur in order to develop strategies for choosing which patients may benefit by adjuvant therapy. In our stage I lung adenocarcinomas we found the histological subtypes proposed according to the new classification fell into three major prognostic categories or grades of behavior. Two categories (adenocarcinoma in situ and MIA) had 100\% 5-year disease-free survival. In the future, such patients may represent candidates for limited surgical resection; however, it can be very difficult for a pathologist to exclude invasion on the basis of frozen section and the role of frozen section in this setting still needs to be defined. ${ }^{8}$ Lepidic, acinar and papillary predominant adenocarcinomas had an intermediate clinical behavior with 5-year disease-free survival between 83 and $90 \%$. Finally, a poor prognostic group consisted of the four categories solid, micropapillary, and colloid predominant as well as invasive mucinous adenocarcinoma with 5-year disease-free survival between 67 and $76 \%$. Not only do these histological subtypes fall into overall prognostic categories or grades of behavior as we demonstrate in this study, but Sica $G$ et al have shown that the detailed data derived from comprehensive histological subtyping appear to be useful for developing a more elaborate grading system for lung adenocarcinoma according to tumor architecture. ${ }^{24}$

In our series, the adenocarcinoma in situ and MIA cases comprised less than $2 \%$, the 29 lepidic predominant adenocarcinomas, comprised $5.6 \%$, and the remaining $93 \%$ of cases represented predominantly invasive adenocarcinomas. These predominantly invasive adenocarcinomas, comprise the largest subset of tumors and presented the greatest challenge of this new classification because they are composed of a complex heterogeneous mixture of the different histological patterns. By using the method of comprehensive histological subtyping, ${ }^{4}$ we were able to classify the tumors according to the predominant subtype and identify prognostically significant categories.

Our data also confirm that cases previously classified as BAC now fall into five different entities in the new IASLC/ATS/ERS classification including (1) adenocarcinoma in situ, (2) MIA, (3) lepidic predominant adenocarcinoma, (4) other invasive adenocarcinomas with lepidic component (this includes patients with advanced lung adenocarcinoma, although these cases were excluded from this study) and (5) mucinous adenocarcinoma. The widely varying clinical behavior of the different categories observed in this study supports the rationale in the new classification for discontinuing use of the term BAC.

The poor prognostic group of solid, micropapillary, invasive mucinous adenocarcinoma and colloid predominant subtypes are important because patients with these tumors may be candidates for adjuvant therapy. There is a growing amount of data confirming that the solid subtype correlates with poor prognosis including a study from our group in a different data set $^{4}$ and data from other investigators. ${ }^{28,29}$ The adverse prognosis associated with the micropapillary pattern, confirms observations in multiple previous studies and supports the addition of this new subtype to the classification. ${ }^{10,11,30-33}$ However, varied criteria for the diagnosis have been used with percentage of the micropapillary component including as little as $6 \%$ of the total tumor and none of these studies have utilized comprehensive histological subtyping to define this subgroup according to the predominant subtype showing comparison with survival of the other histological subtypes as we have done. ${ }^{10,11,30-33}$ Our finding that colloid adenocarcinomas also fit into this poor prognostic group was somewhat surprising as these tumors are often regarded as well-differentiated tumors, but this is the largest series of these tumors reported where survival analysis compared outcome with other adenocarcinoma histological types. Furthermore, no previous studies have ever defined colloid adenocarcinomas according to the predominant subtype.

One of the most significant changes in the new classification is separation of the previous mucinous BACs into a separate category called invasive mucinous adenocarcinoma. This is based not only on the different histological appearance, but the frequent presentation with multiple nodules, lobar consolidation, multilobar and/or bilateral lung involvement and frequent KRAS mutations compared with EGFR mutations. ${ }^{8}$ Worse survival for tumors formerly classified as mucinous BAC compared with those formerly classified as nonmucinous BAC has been suggested. ${ }^{18,34-36}$ However, the data are limited, other studies have not shown a significant survival difference, ${ }^{37}$ and the histological criteria have evolved over time. ${ }^{26}$ The latter problem complicates interpretation of the literature on this topic. Our finding that invasive mucinous adenocarcinoma fits with the high-grade clinical behavior is consistent with the previous literature suggesting an aggressive clinical course for these tumors. ${ }^{34,35,38}$ This is consistent with our finding in this study that most tumors previously classified as mucinous BACs actually show invasive growth rather than a pure lepidic pattern.

Our data also raise the question whether T-factor staging for tumor size should be based only on the invasive component rather than total tumor size, in a manner similar to breast cancer wherein the size of the in situ component is not included in T-factor staging. ${ }^{39}$ This new way of thinking was a direct result of introduction of the concept of adenocarcinoma in situ for small solitary lung adenocarcinomas with pure lepidic growth. Despite the slightly stronger association with survival for invasive size 
versus gross size $(P=0.001$ versus 0.04$)$, in our data set, both approaches appeared to demonstrate prognostic significance for survival. The potential for this to impact staging for small lung adenocarcinomas needs further evaluation to see whether this could impact the next revision of TNM staging. Another implication for staging is to use the term Tis (adenocarcinoma) in distinction from Tis (squamous) for lung cancer that meet criteria for adenocarcinoma in situ, just as in breast cancer Tis (ductal carcinoma in situ) and Tis (lobular carcinoma in situ). ${ }^{39}$ Regarding MIA, again similar to breast cancer, a new category of Tmi may be introduced for lung adenocarcinomas that meet criteria for this new proposed category. ${ }^{39}$ As the 7 th edition of the Union for International Cancer Control/American Joint Committee on Cancer staging system was just recently published, ${ }^{22,23}$ these topics will need to be the focus of investigation for the next 5 years so that sufficient validated data is available to determine which changes need to be made in the 8th edition of the TNM classification for lung adenocarcinoma staging.

Another observation in our study was that disease-free survival was more informative than overall survival in predicting outcome in these stage I adenocarcinomas. Overall survival is the method of survival analysis utilized in most lung cancer studies. This is because the high mortality of lung cancer diminishes the impact of the small percentage of patients who die of other causes. However, in tumor populations such as our series of stage I adenocarcinomas, where there is a high percentage of deaths not related to tumor, disease-free survival more accurately reflects the biological behavior of the tumors.

In summary, our study of stage I lung adenocarcinomas demonstrates the new IASLC/ATS/ERS classification identifies prognostically significant histological subtypes of lung adenocarcinomas including the low-grade adenocarcinoma in situ and MIA and the intermediate grade lepidic predominant, acinar and papillary adenocarcinomas. High-grade adenocarcinoma subtypes included solid, micropapillary, invasive mucinous adenocarcinoma and colloid predominant adenocarcinomas. Thus histological subtyping of adenocarcinomas is a potential a way to stratify patients with stage I tumors for adjuvant therapy.

\section{Acknowledgement}

We gratefully acknowledge the assistance of Kei Suzuki and Kyuichi Kadota in analyzing the pleura for visceral pleural invasion.

\section{Disclosure/conflict of interest}

The authors declare no conflict of interest.

\section{References}

1 Boyle P, Levin B. World Cancer Report 2008. International Agency for Research on Cancer: Lyon, 2008.

2 Pao W, Miller V, Zakowski M, et al. EGF receptor gene mutations are common in lung cancers from 'never smokers' and are associated with sensitivity of tumors to gefitinib and erlotinib. Proc Natl Acad Sci USA 2004;101:13306-13311.

3 Mok TS, Wu YL, Thongprasert S, et al. Gefitinib or carboplatin-paclitaxel in pulmonary adenocarcinoma. N Engl J Med 2009;361:947-957.

4 Motoi N, Szoke J, Riely GJ, et al. Lung adenocarcinoma: modification of the 2004 WHO Mixed subtype to include the major histologic subtype suggests correlations between papillary and micropapillary adenocarcinoma subtypes, EGFR mutations and gene expression analysis. Am J Surg Pathol 2008;32: 810-827.

5 Noguchi M, Morikawa A, Kawasaki M, et al. Small adenocarcinoma of the lung. Histologic characteristics and prognosis. Cancer 1995;75:2844-2852.

$6 \mathrm{Kim}$ EA, Johkoh T, Lee KS, et al. Quantification of ground-glass opacity on high-resolution CT of small peripheral adenocarcinoma of the lung: pathologic and prognostic implications. AJR Am J Roentgenol 2001; 177:1417-1422.

$7 \mathrm{Wu}$ J, Ohta Y, Minato H, et al. Nodal occult metastasis in patients with peripheral lung adenocarcinoma of $2.0 \mathrm{~cm}$ or less in diameter. Ann Thorac Surg 2001;71:1772-1777.

8 Travis WD, Brambilla E, Noguchi M, et al. The new IASLC/ATS/ERS international multidisciplinary lung adenocarcinoma classification. J Thoracic Oncol 2011; 6:244-285.

9 Tsutsumida H, Nomoto M, Goto M, et al. A micropapillary pattern is predictive of a poor prognosis in lung adenocarcinoma, and reduced surfactant apoprotein A expression in the micropapillary pattern is an excellent indicator of a poor prognosis. Mod Pathol 2007;20:638-647.

10 Miyoshi T, Satoh Y, Okumura S, et al. Early-stage lung adenocarcinomas with a micropapillary pattern, a distinct pathologic marker for a significantly poor prognosis. Am J Surg Pathol 2003;27:101-109.

11 Amin MB, Tamboli P, Merchant SH, et al. Micropapillary component in lung adenocarcinoma: a distinctive histologic feature with possible prognostic significance. Am J Surg Pathol 2002;26:358-364.

12 Gaeta M, Blandino A, Pergolizzi S, et al. Patterns of recurrence of bronchioloalveolar cell carcinoma after surgical resection: a radiological, histological, and immunohistochemical study. Lung Cancer 2003;42: 319-326.

13 Marchetti A, Buttitta F, Pellegrini S, et al. Bronchioloalveolar lung carcinomas: K-ras mutations are constant events in the mucinous subtype. J Pathol 1996;179:254-259.

14 Yatabe Y, Koga T, Mitsudomi T, et al. CK20 expression, CDX2 expression, K-ras mutation, and goblet cell morphology in a subset of lung adenocarcinomas. J Pathol 2004;203:645-652.

15 Goldstein NS, Thomas M. Mucinous and nonmucinous bronchioloalveolar adenocarcinomas have distinct staining patterns with thyroid transcription factor and cytokeratin 20 antibodies. Am J Clin Pathol 2001;116:319-325. 
16 Garfield DH, Cadranel J. The importance of distinguishing mucinous and nonmucinous bronchioloalveolar carcinomas. Lung 2009;187:207-208.

17 Lee HY, Lee KS, Han J, et al. Mucinous versus nonmucinous solitary pulmonary nodular bronchioloalveolar carcinoma: CT and FDG PET findings and pathologic comparisons. Lung Cancer 2009;65: 170-175.

18 Wislez M, Antoine M, Baudrin L, et al. Non-mucinous and mucinous subtypes of adenocarcinoma with bronchioloalveolar carcinoma features differ by biomarker expression and in the response to gefitinib. Lung Cancer 2010;68:185-191.

19 Finberg KE, Sequist LV, Joshi VA, et al. Mucinous differentiation correlates with absence of EGFR mutation and presence of KRAS mutation in lung adenocarcinomas with bronchioloalveolar features. J Mol Diagn 2007;9:320-326.

20 Cohen PR, Yoshizawa A, Motoi N, et al. Signet ring cell features (SRCF) in lung adenocarcinoma: a cytologic feature or a histologic subtype? Mod Pathol 2010;23.

21 Deshpande CG, Yoshizawa A, Motoi N, et al. Clear cell change in lung adenocarcinoma: a cytologic change rather than a histologic variant. Mod Pathol 2009;22.

22 Goldstraw P. IASLC Staging Manual in Thoracic Oncology 1st edn. International Association for the Study of Lung Cancer, Editorial Rx Press: Orange Parka, FL, 2009.

23 Rusch VW, Appleman HD, Blackstone E, et al. Lung In: Edge SB, Byrd DR, Compton CC, Fritz AG, Greene FL, Trotti A (eds). AJCC Cancer Staging Manual 7th edn. American Joint Commision on Cancer, Springer: Chicago, 2009, pp 253-270.

24 Sica G, Yoshizawa A, Sima CS, et al. A grading system of lung adenocarcinomas based on histologic pattern is predictive of disease recurrence in stage I tumors. Am J Surg Pathol 2010;34:1155-1162.

25 Travis WD, Brambilla E, Rami-Porta R, et al. Visceral pleural invasion: pathologic criteria and use of elastic stains: proposal for the 7th edition of the TNM classification for lung cancer. J Thorac Oncol 2008; 3:1384-1390.

26 Travis WD, Brambilla E, Müller-Hermelink HK, et al. Pathology and Genetics: Tumours of the Lung, Pleura, Thymus and Heart Vol 1 IARC: Lyon, 2004.

27 Goldstraw P, Crowley J, Chansky K, et al. The IASLC lung cancer staging project: proposals for the revision of the TNM stage groupings in the forthcoming (seventh) edition of the TNM Classification of malignant tumours. J Thorac Oncol 2007;2:706-714.

28 Barletta JA, Yeap BY, Chirieac LR. Prognostic significance of grading in lung adenocarcinoma. Cancer 2010;116:659-669.

29 Riquet M, Foucault C, Berna P, et al. Prognostic value of histology in resected lung cancer with emphasis on the relevance of the adenocarcinoma subtyping. Ann Thorac Surg 2006;81:1988-1995.

30 Nagano T, Ishii G, Nagai K, et al. Structural and biological properties of a papillary component generating a micropapillary component in lung adenocarcinoma. Lung Cancer 2010;67:282-289.

31 Kamiya K, Hayashi Y, Douguchi J, et al. Histopathological features and prognostic significance of the micropapillary pattern in lung adenocarcinoma. Mod Pathol 2008;21:992-1001.

32 Kawakami T, Nabeshima K, Makimoto Y, et al. Micropapillary pattern and grade of stromal invasion in pT1 adenocarcinoma of the lung: usefulness as prognostic factors. Mod Pathol 2007;20:514-521.

33 Makimoto Y, Nabeshima K, Iwasaki H, et al. Micropapillary pattern: a distinct pathological marker to subclassify tumours with a significantly poor prognosis within small peripheral lung adenocarcinoma $(</=20 \mathrm{~mm})$ with mixed bronchioloalveolar and invasive subtypes (Noguchi's type C tumours). Histopathology 2005;46:677-684.

34 Daly RC, Trastek VF, Pairolero PC, et al. Bronchoalveolar carcinoma: factors affecting survival. Ann Thorac Surg 1991;51:368-376.

35 Manning Jr JT, Spjut HJ, Tschen JA. Bronchioloalveolar carcinoma: the significance of two histopathologic types. Cancer 1984;54:525-534.

36 Cadranel J, Quoix E, Baudrin L, et al. IFCT-0401 Trial: a phase II study of gefitinib administered as first-line treatment in advanced adenocarcinoma with bronchioloalveolar carcinoma subtype. J Thorac Oncol 2009;4:1126-1135.

37 Garfield DH, Cadranel J, West HL. Bronchioloalveolar carcinoma: the case for two diseases. Clin Lung Cancer 2008;9:24-29.

38 Clayton F. Bronchioloalveolar carcinomas. Cell types, patterns of growth, and prognostic correlates. Cancer 1986;57:1555-1564.

39 Hayes DF, Allred C, Anderson BO, et al. Breast. In: Edge SB, Byrd DR, Compton CC, Fritz AG, Greene FL, Trotti A (eds), AJCC Cancer Staging Manual, 7th edn, Springer: New York, 2009. 